\title{
Correspondence
}

Psychological Medicine, 46 (2016).

doi:10.1017/S0033291715002135

\section{Research Letter \\ PTSD has shared polygenic contributions with bipolar disorder and schizophrenia in women}

\section{Introduction}

Twin studies have demonstrated overlap between genetic contributions to post-traumatic stress disorder (PTSD) and other psychiatric disorders (Kremen et al. 2012). These findings have prompted interest in examining shared genetic risk between PTSD and other psychopathology at the molecular level. With genomewide association studies (GWAS) and collaborative consortia-based efforts, replicable risk variants have been identified for schizophrenia and bipolar disorder (Cross-Disorder Group of the Psychiatric Genomics Consortium, 2013; Schizophrenia Working Group of the Psychiatric Genomics Consortium, 2014). Analyses of genetic loci in aggregate (polygenic effects; Purcell et al. 2009) have demonstrated shared genetic risk between schizophrenia, bipolar disorder, and major depressive disorder (MDD), with greatest overlap for schizophrenia and bipolar disorder (Cross-Disorder Group of the Psychiatric Genomics Consortium, 2013). Using 3742 candidate single nucleotide polymorphisms (SNPs), an initial polygenic analysis of PTSD by our group (Solovieff et al. 2014) suggested overlap in genetic risk for bipolar disorder and PTSD in European American (EA) women that was replicated in a male EA sample with genome-wide data (Nievergelt et al. 2015).

\section{Method}

Here we extend our previous investigation (Solovieff et al. 2014) by examining associations between polygenic scores computed on genome-wide data using results from the Psychiatric Genomics Consortium (PGC) for bipolar disorder, MDD, and schizophrenia (the discovery samples) with PTSD in 1293 traumaexposed EA women in the PTSD diagnostic subsample of the Nurses' Health Study II (the target sample). Interviews assessed participants' trauma history and the 17 DSM-IV PTSD symptoms subsequent to their worst trauma. All procedures contributing to this work comply with the ethical standards of the relevant national and institutional committees on human experimentation and with the Helsinki Declaration of 1975 , as revised in 2008. Forty-four percent $(n=563)$ of women met PTSD criteria; mean PTSD severity score (calculated by summing responses to the 17 symptoms) was 32.3 (S.D. $=14.5$; range $=17-85$ ). Mean age at study baseline was 35.9 (s.D. $=4.3$; range $=24-43$ ).

DNA was extracted from blood samples. Genotyping was performed with the Illumina Infinium PsychArray BeadChip (Psych Chip), which assesses $500000+$ psychiatric-relevant markers genome-wide. Standard GWAS quality control, phasing, and imputation procedures were performed as in the PGC Schizophrenia Working Group (Schizophrenia Working Group of the Psychiatric Genomics Consortium, 2014). Overlap between previously examined SNPs in this sample (Solovieff et al. 2014) and the genome-wide data was minimal. Using the PGC polygenic score approach (Purcell et al. 2009), we computed polygenic scores for bipolar disorder, MDD, and schizophrenia based on linkage disequilibrium-pruned results from the largest available studies of these disorders (http://www.med.unc.edu/pgc/ downloads). For each disorder, polygenic scores at varying $p$ value thresholds were computed by summing the number of risk alleles for a participant weighted by the natural $\log$ of the odds ratio for each SNP. Polygenic scores were computed in PLINK 1.9 (https://www.cog-genomics.org/ plink2). The first 10 principal components from a principal components analysis were covaried in analyses.

\section{Results}

Logistic and linear regressions predicting PTSD diagnosis and severity, respectively, from the polygenic scores demonstrated overlap in common genetic risk for bipolar disorder and schizophrenia with PTSD (Table 1). Associations generally became stronger with more liberal $p$ value thresholds. As is typical in polygenic score analyses (Solovieff et al. 2014; Nievergelt et al. 2015), nominal significance was set at $p<0.05$, although we note that some associations with the schizophrenia-based scores survived Bonferroni correction $(p<0.0014)$ - a highly conservative approach given that many tests were correlated. Bipolar disorder and schizophrenia polygenic scores accounted for a small percentage $(<1.2 \%)$ of the variance in PTSD outcomes (Table 1). No significant associations emerged for MDD-based scores.

\section{Discussion}

Consistent with prior research (Solovieff et al. 2014; Nievergelt et al. 2015), our findings suggest that 
common genetic variants for bipolar disorder index genetic risk for PTSD in women. We extended these previous findings by further demonstrating significant overlap between polygenic scores for schizophrenia and PTSD. Effects were small but consistent with others in the literature (Cross-Disorder Group of the Psychiatric Genomics Consortium, 2013) and with the notion of shared genetic vulnerability across diagnostic categories. The lack of significant results for MDDbased scores is consistent with two previous reports (Solovieff et al. 2014; Nievergelt et al. 2015). Although surprising given genetic overlap between MDD and PTSD in twin studies (Kremen et al. 2012), the results parallel the underpowered PGC MDD GWAS, which has no significant loci to date (Cross-Disorder Group of the Psychiatric Genomics Consortium, 2013). Additional research needs to assess generalizability of findings and whether results reflect unique bipolarPTSD and schizophrenia-PTSD variants or genetic variation associated with general psychopathology risk.

\section{Acknowledgements}

This study was supported by the National Institutes of Health grants R01 MH078828 (to Dr. Koenen), U01 MH094421 (for development of the Psych Chip), and UM1 CA176726 (for NHS II infrastructure). We acknowledge the Channing Division of Network Medicine, Department of Medicine, Brigham and Women's Hospital, and Harvard Medical School for managing the NHS II.

\section{Declaration of Interest}

None.

\section{References}

Cross-Disorder Group of the Psychiatric Genomics Consortium (2013). Identification of risk loci with shared effects on five major psychiatric disorders: a genome-wide analysis. Lancet 381, 1371-1379.

Kremen WS, Koenen KC, Afari N, Lyons MJ (2012). Twin studies of posttraumatic stress disorder: differentiating vulnerability factors from sequelae. Neuropharmacology $\mathbf{6 2}$, 647-653.

Nievergelt CM, Maihofer AX, Mustapic M, Yurgil KA, Schork NJ, Miller MW, Logue MW, Geyer MA, Risbrough VB, O'Connor DT, Baker DG (2015). Genomic predictors of combat stress vulnerability and resilience in U.S. Marines: a genome-wide association study across multiple ancestries implicates PRTFDC1 as a potential PTSD gene. Psychoneuroendocrinology 51, 459-471.

Purcell SM, Wray NR, Stone JL, Visscher PM, O'Donovan MC, Sullivan PF, Sklar P (2009). Common polygenic 
variation contributes to risk of schizophrenia and bipolar disorder. Nature 460, 748-752.

Schizophrenia Working Group of the Psychiatric Genomics Consortium (2014). Biological insights from 108 schizophrenia-associated genetic loci. Nature 511, 421-427.

Solovieff N, Roberts AL, Ratanatharathorn A, Haloosim M, De Vivo I, King AP, Liberzon I, Aiello A, Uddin $M$, Wildman DE, Galea S, Smoller JW, Purcell SM, Koenen KC (2014). Genetic association analysis of 300 genes identifies a risk haplotype in SLC18A2 for post-traumatic stress disorder in two independent samples. Neuropsychopharmacology 39, 1872-1879.

J. A. SUMNER ${ }^{1,2, *}$, L. DUNCAN ${ }^{3,4,5}$, A. RATANATHARATHORN ${ }^{2,6}$, A. L. ROBERTS AND K. C. KOENEN $2,3,8$

${ }^{1}$ Center for Behavioral Cardiovascular Health, Columbia University Medical Center, New York, NY, USA

${ }^{2}$ Department of Epidemiology, Harvard T. H. Chan School of Public Health, Boston, MA, USA
${ }^{3}$ Stanley Center for Psychiatric Research, Broad Institute of MIT and Harvard, Cambridge, MA, USA

${ }^{4}$ Analytic and Translational Genetics Unit, Psychiatric and Neurodevelopmental Genetics Unit, Massachusetts General Hospital, Boston, MA, USA

${ }^{5}$ Medical and Population Genetics Program, Broad Institute of MIT and Harvard, Cambridge, MA, USA

${ }^{6}$ Department of Epidemiology, Columbia University Mailman School of Public Health, New York, NY, USA ${ }^{7}$ Department of Social and Behavioral Sciences, Harvard T. H. Chan School of Public Health, Boston, MA, USA ${ }^{8}$ Psychiatric and Neurodevelopmental Genetics Unit and Department of Psychiatry, Massachusetts General Hospital, Boston, MA, USA

*Author for correspondence: J. A. Sumner, PhD, Center for Behavioral Cardiovascular Health, Columbia University Medical Center, 622 W.168th St, PH 9-315, New York, NY 10032, USA.

(Email: js4456@cumc.columbia.edu) 